\title{
Amélioration des conditions d'écoulement dans les turbines Francis
}

\author{
Description sommaire et effets des "pointes perforées stabilisatrices"
}

\section{Improvement of the flow conditions in Francis turbines Brief description and effect of "perforated stabilising caps"}

\author{
E. FONTAINE
}

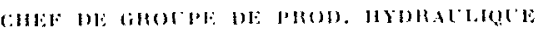

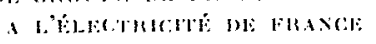

PAR

\section{PEYRIN}

MCENIETH

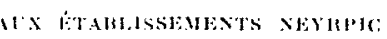

\author{
(i. WIILII
}

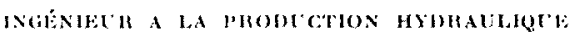

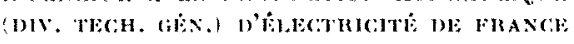

\begin{abstract}
líbonlement a In sortie de rones de larbines hydranliques indastrielles a réaction est soubont assez bloigne de re gue montrent des essais rfeclues en similitude partielle sur modele réduit. el plas particulierement lorsque les condilions earctes de cavitation n'ont pas ife introduites en similitude roviecte; des regimes critiques, des pulsations, des pibrations on des chocs, prennent natssance dans les aspirateurs, en liaison aber des phenomines plas ot moins acentue's de ravitalion. Ces manifestations sibl non seulement bruyantes, mais encore pal faborables a la bonno conservalion des differents organes des turbines et méme des allemalears. He flus, "lles laissent présuger des pertes d'inergie el des baisses de rendement. hes essais in silu effectuas dis last it Brommat pror MM. Fonlaine el Campmas, ingénieurs delerdricile de France, lear ont permis d'explorer la rhamp des nitesses dans l'uspirateur

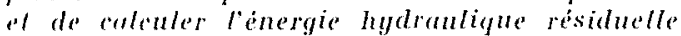
a la sortie de la rone.

Les résullats de res ifudes ont amene H. Fonlaine it rechercher l'améliombion de l'aronlement, soit pat des modifications dans lo trace de sortie des anbages, soil en collaboralion aber M. Peyrin, ingenieur anx Etablissements Neprpic, par la reralion el la mise all poiml des pointes perforés stabilisatrices.

Des essuis récents effechus a lasine de Saintreniez-b-Herle ont montre le gatin sensible de rendement at le paissance qui en risulle.
\end{abstract}

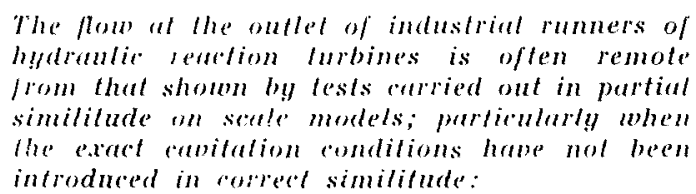

Gritical regimes, pulsations, mibrolions or shocks aome to life in the draft lubes logether with some'imes important coutation phenomena. These ocourrences ate not only noisy. but then are as mell anfueourable to the preserantion of the larbine parts and enen of the allernetors. They also orension chergy losses and louser ontpril.

In siln tests iamied oul since $195 \mathrm{~J}$ at Brommal by Messs. Fonlaine and Campmas, Fngibres of Elerlricite de France. hane enabled them to cormine tho belocity distribution in the drat lube and ta raldulate the residual hydratio energ!l al the runuer antlet. The resulls of these studies hame led Y. Fontaine lo look for impromements to the fiou eiller by modifying the form of the omlled from the blades, of to-

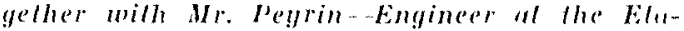
blissements Negrpir-by the design and applicration of perforated stabilising caps.

Recent lests atried nut al lhe Saint-leniezio-kele pomer station hane sheswn the apprerinble gains in efficienc!l and poner mhich resull.

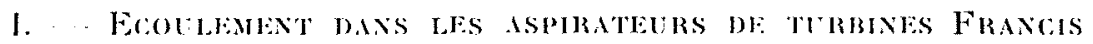

L'étude de lécoulement dans les aspiraleurs de lurbines Francis, commencée à Brommat en 1951, et basée sur la mesure des pressions el des ritesses a la sortie de la roue, a permis de définir la notion d'inergie hydraulique résiduelle, encrgic par définition non récupérable et donc équivalente à une perte. On démontre (") que cette 
Energic, qui a la dimension d'une hauleur de chute, a pour forme :

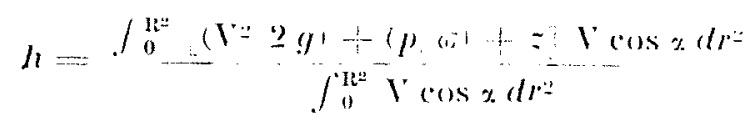

Vétant la vilesse de l'ean a la sortie de la roue a tune distance $r$ do l'axe, $\neq$ l'angle de cotle vitesse avec l'axe de l'éconlement el z la différance de cole entre le point de mesure et le niveau aval.

Praliquement, les vilesses varienl sensiblement en grandeur et en direction suivant l'abscisse $r$ du point considéré el la charge de la turbine. L'angle $x$, en particulier, peut prendre avec corlains traces d'aubage des valeurs importantes el devient même voisin de 90" dans la zone centrale de l'écoulement qui prend l'allure d'un pseudo-vortex dénomme \& torche ou « trombe ». La quasi-totalité du débit turbine se concentre alors dans la zone annulaire comprise entre la torche et la paroi de l'aspirateur: les vitesses y sont tres élevées et le fonclionmement de l'aspirateur en recupérateur de $\mathrm{V}: 2 \mathrm{~g}$ fortement compromis. En fail, les essais dont il a été parlé plus haut ont confirmé qu'ume parl importante des pertes de la turbine etail représentée par celle énergie non récupéréc à la sortic de la roue.

Par ailleurs, les phenomenes transitoiares (chocs, vibrations, etc.) peuvent etre attribues à l'inslabilite de forme du pseudo-vortex dont la foi de rotation, il faut le noter, differe sensiblement de celle du vortex mathematique : du fait des forces tangentielles de viseosité, la «torehe» lourne en bloc, les vitesses circonférenliclles sonl proportionnelles au rayon).

La combinaison des mouvements de celle * torehe $》$ et de léeoulement emportaml la part d'énergie aval non récupéréc dome un ensemble présentant un balourd de masse suffisamment important pour ébranler les cones d'aspirateurs, an mene temps que se lait entendre un grondement rythmé caractéristique. On établit et on constate que la torche lourne dans le sens de la roue aux faibles admissions, el en sens inverse aux forles charges, ce qui se lraduil dans le hief aval par le déport de l'écoulement vers une rive ou vers !'autre.

Enlre ces deux tranches d'admission, existe tune tranche intermédiaire en gineral situce au voisinage des rendements maxima, ou les régimes de rotation sonl complexes et où se produisent les plus forls a-coups de cavitation cenIrale : on peut penser que la circulation induile par la roue dans le noyau du pseudo-vortex n'est pas suffisamment affirmée pour stabiliser celui-ei.
On a pensé que les rendements déja bons correspondant a cette tranche d'utilisation pourraient c̀tre élales, et mème augmentes, si les perturbations centrales etaient atténueses.

Il est connu que la violence des perturbations peut ètre atlénuée par une introduction d'air sous ia roue, en particulier au centre de l'ecoulement. Les dispositifs d'acration, somvent complexes, mais necessaires pour assurer aux grands groupes hydroélectriques un fonctionnement mécanique satisfaisant, ne constituent qu'un palliatid ni tris élégant, ni tres technique.

Au conlraire, un dispositif organique laisant parlie intégrante de la roue de la furbine apparail bien preférable; on demandera a ce dispositif de stabiliser les icoulements, de reduire les espaces turbulents, de detruire an moins partiellement la torche el d'amortir les choes de cavilation.

Un tel probleme itail particulicrement urgent à résoudre à Chastang, hant en raison de limportance des vibrations qui se transmellaient jusqu a la pivolerie que de l'existence de charges criligues incompatibles avec les hesoins de l'exploitation. A cetle oceasion, la collahoration de M. PEYmis, des Etablissements Neyrpic, of de M. Foncaive, Chef de ciroupe de Production Hydratique de l'Electricité de France, a clé à l'origine de l'invention du dispositif dit \& pointe stabilisatries perforie $\$\left({ }^{\star}\right.$ ); grâce au concours de lout le personnel du Groupe de Production, leur mise au point a pu etre ellectué en quelques mois. Il y a lieu de signaler Lout de suile que l'elfet de ees "pointes perforees sur les imporlantes turbines de 134.000 ch sous 70 m de chule à Chastang a ele des plus inleressants : les vibrations el charges critiques onl completement disparu, l'écoulement a la sortic des pertuis de laspiraleur a de lranquillise, les machines sont devenues stables a loutes les charges.

\section{DESCRIPTION D'UNE POINTH STABHISATRICE PERFOMÉ:}

La figure n" I montre l'importance des forutes geomchlofues de la pointe stabilisalrice patr rapport au guideau de fables proportions qui prolongeail aulrefois les plafonds des roues. Le pied émousse de edle pointe doit se siluer assez en aral de la sortie des aubages atin que son effel s'exerce dans celle zone que nous disons etre la zone de transition entre la sortie de la roue et la naissance de l'aspirateur.

\footnotetext{
1.) Les brevels Foxtane-levan pris an forance ef dans tous les pays etrangers el laurs alditifs semon exploites en commun pat E.D.F. et Neypic.
} 
Ce pied de pointe est perforé d'un grand nombre de trous qui lui donnent l'aspect d'une cripine. A l'autre extrémité de la pointe, vers la grande base du tronc de còne, sont percés plusieurs rangs circonférentiels de trous; le premier de ces rangs doit être situé près de la sortie des aubes.

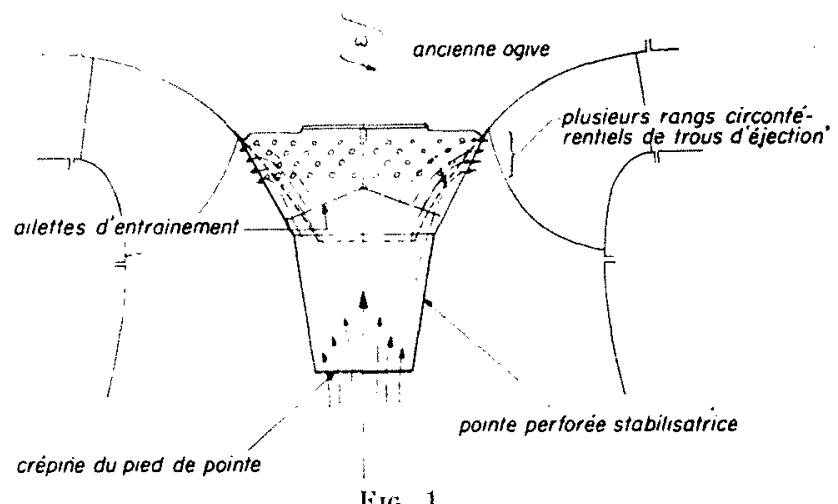

Fig. 1

A l'intérieur de la pointe et à hauteur des trous circonférentiels peuvent, dans certains cas, être disposées des nervures ou ailettes diamétrales qui ont pour effet d'entrainer l'eau contenue dans la pointe en rotation à la mème vitesse que la turbine.

\section{III. - ACTION DE LA pOINTE PERforÉE SUR L'ÉCOLLHMENT APHÉs LA BOUE}

Dans la rotation, les trous supérieurs circonlérentiels éjectent de l'eau par effet centrifuge; li grille de pied admet évidemment le même débit. On appellera les rangs circonférentiels de trous vers la grande base des cônes «trous d'éjection » »et la crépine inférieure « crépine d'aspiration $»$.

La crépine, due à M. Fontarne, introduite at cours de l'expérimentation, qu'il a conduite à Chastang, est apparue l'un des éléments très importants de la mise au point de la pointe stabilisatrice; elle s'est trouvíe en relation s'troite et fayorable d'eflet avec la perforation circonférenlielle d'éjection de la grande base.

Sur la figure 2, on a représenté pour fixer les idíes une lurbine et son écoulement schématisé pour une faible charge; les frontières de la zone vive d'écoulement sont constituées d'une part par le llasque inférieur de la turbine, continué par la ceinture de rote, puis plus bas par les parois du tuyau d'aspiration; d'autre part, par une surface mcridienne d'écoulement $a b c$.

On a représenté schématiquement entre ces deux surfaces limites quelques traces des composantes méridiennes du vif de l'écoulement. A l'in- lérieur du noyau de diamètre $\mathrm{N}$ existe l'espace mort qui est préjudiciable au bon fonctionnement de la machine. Ce noyau est le siège de rotations induites par l'écoulement périphérique (voir plus haut).

Il est encore essentiel de considérer les courants secondaires, schématisés par des lignes munies de fleches, s'enroulant en constituan! dans leur ensemble autour de l'axe de rotation ir la turbine comme un anneau tourbillon dont

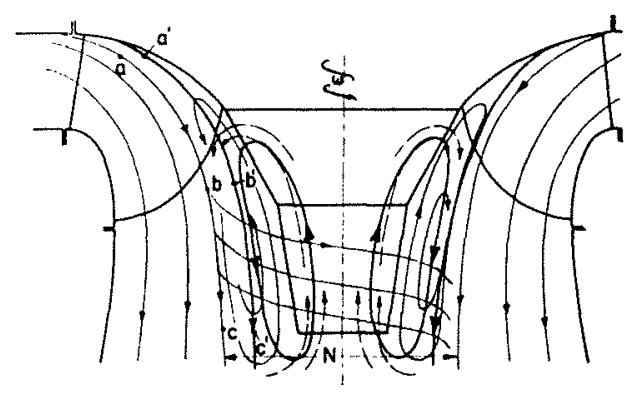

Fig. 2

le sens de rolation résulte entre autres du sens de l'écoulement méridien du flux actif.

Cet anneau tourbillon (aspect bien connu d'un amneau de fumée) épousait au milieu, dans sa remonte vers les aubes, la forme du guideau central que constituait l'ancienne pointe.

L'un des effets de la pointe est de fixer la circulation de cet anneau tourbillon autour de sa paroi, comme schématisé sur la figure 2, dans un cas particulier le plus souvent constaté.

\section{IV. -... Constequnces \\ DES MODHFICATIONS DES FORMLS DE L'ÉCOULEMENT IUSCLTANT \\ DE IA PHÉSENCE DE LA POINTE PERFORÉE}

La réduction de Ia « torche», l'atténuation de son mouvement torse de balayage nuisible $\mathrm{du}$ flux utile, l'élargissement de l'espace octroyé it celui-ci et la stabilisation de ses formes dans la zone où commence la transformation de l'énergie cinétique en énergie potentielle, a comme consćquence éminemment favorable la régularisation du champ des vitesses.

Dans l'ensemble, le champ des énergies se présente sous une forme améliorée, qui se prête vraisemblablement mieux à la récupération de l'énergie cinétique disponible à la sortie de la roue.

L'effet de l'aspirateur s'appliquant alors à une énergie plus docile ne peut qu'être de meilleure efficience. Il est déjà reconnu que l'écoulement, dans son ensemble, répond mieux à la notion de continuité, et que les vibrations pernicieuses 

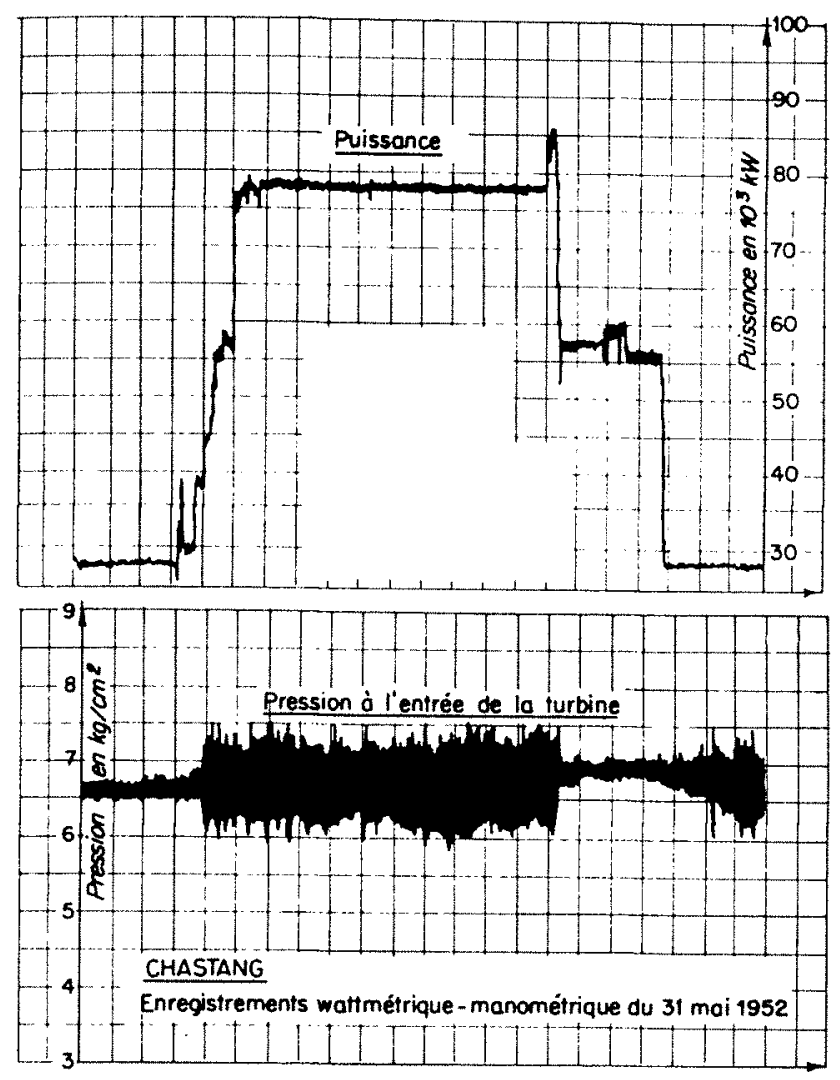

Fig, 3

Ondes entretenues de pression arec pointe de roue ordinaire

(aves insufflation d'air, $5 \mathrm{~m}$ : $: m 11$ ),

ainsi que les fortes détonations disparaissent presque completement (roir fig. 3 et 4 ).

Des collines de laboratoire montrent que les rendements sont améliorés et étalés dans la zone où ils étaient déjà satisfaisants, mạis perlurbés par les instabilités.

Il devient possible d'exploiter sans introduction d'air des turbines, qui, sans la pointe terminale spéciale, en nécessitaient en permanence un débil très strand; ainsi l’on peut déjà, de ce fait, escompter une amélioration de rendement de récupération, indépendamment du gain venant des meilleures formes de l'écoulement ion verra au paragraphe VII les résultats oblenus sur des machines en service).

\section{V. - AUTRES EFEETS}

DE: LA POINTE STABITSATHCE PJRFORES

Selon le réglage que l'on fait des orifices éjecteurs par rapport anx orifices aspirateurs, il s'établit à l'intérieur de la pointe une dépression rui peul etre importante, surtout si déjà régne autour d'elle une dépression de récupération déjà forte.

Cette dépression interne accentuée a pour effet de répartir la succion de façon assez uniforme sur toute la crépine de pied; le llux pompé conslitué d'eau et des gaz déjà en partie libérés (qui nourrissaient auparavant le noyau central dans la phase où il grossissait pour atteindre les dimensions critiques instables), divisé à l'extrème dans sa traversée de la multitude des trous de la crépine, se trouve placé dans des conditions physiques de dépression qui favorisent encore le dégazage.

L'intérieur de la pointe est done empli d'une émulsion dont l'élasticité est favorable à labsorption des ondes de pression.

Par ailleurs, l'émulsion éjectée en tête de l'anneau tourbillon parasite schématisé sur la ligure 2, joue aussi le ròle d'amortisseur précisiment dans une zone oul l'action d'une correction, mème de faible importance, peut néanmoins itre très sensible dans l'atténuation de phénomènes violents.

L'ensemble de ces milieux amortisseurs situcs au corur de la rouc, empêche l'aspirateur de vibrer, la propagation d'ondes entretenues plus ou moins stationnaires devenant impossible du fait de la suppression des fronts durs de réllexion.
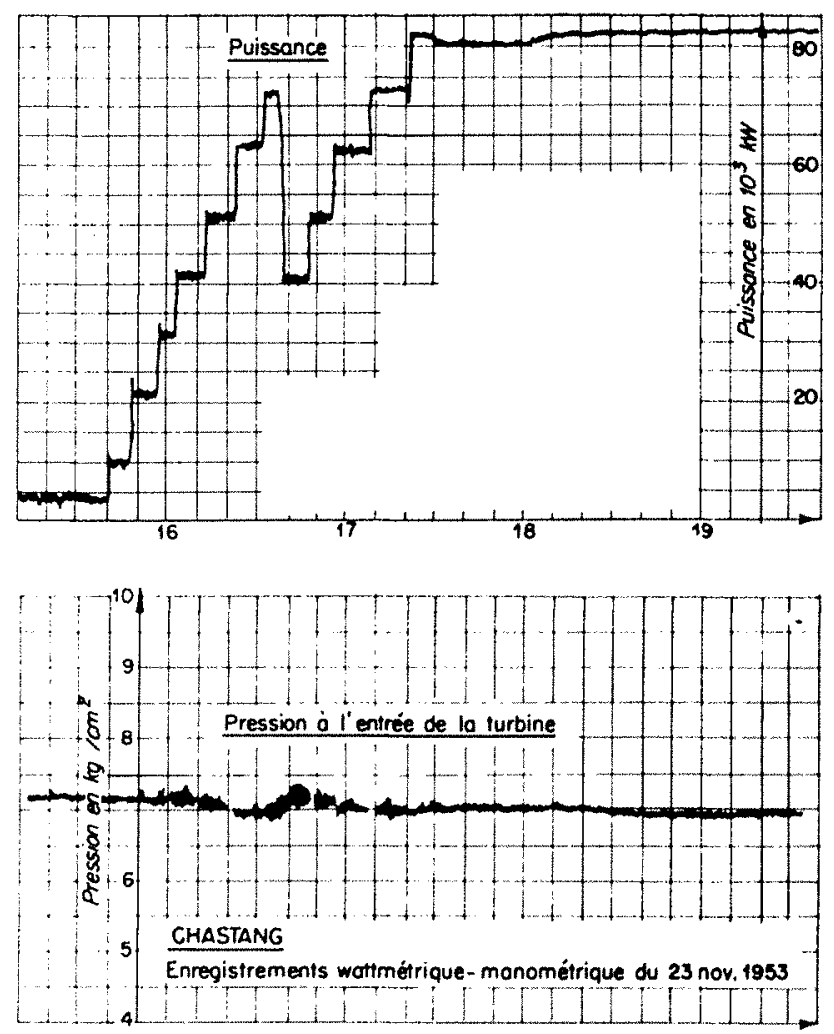

Fin. 4. ... Pointe speciale brevetéc inatrehe sans insurtation d'air). 
On peut encore supposer que l'énergie du dihit éjecté en tête de la circulation parasite troublant les faibles charges, contribue par soulllage a en stabiliser ou détruire les effets néfastes.

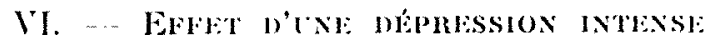 SOUS LA CREPINE DE PIED}

Certaines rotes, lorsqu'elles fonctionnent en surcharge, crécnt à la sortie de leur aubage, côte moyeu, un pseudo-vortex accentué dans le noyau luquel existe alors une dépression anormalement élevée. Fn ce cas, l'effet d'éjection peut etre vaincu par l'efret de succion du vortex; la pointe ne pompe plus dans le sens normal. Les orifices d'éjection deviennent orifices d'aspiration, et leur action dans ce sens peut encore ètre efficace pour contribuer à la stabilité et à la réduction des espaces nuisibles.

\section{VII. - Réstlitas ontenus \\ A SAINT-GENIEz-ô-MrRte}

Cetle usine est équipere de deux groupes à axe vertical d'environ $20.000 \mathrm{~kW}$ mis en service en 1947. La chute brute esl de lordre de $125 \mathrm{~m}$, la vitesse de rotation $375 \mathrm{l} / \mathrm{mn}$.

Les roues originellement identiques ont subi lepuis leur mise en service une série de modilications; de plus, l'une d'elles est équipée d'une " pointe perforée" amovible.

Parmi les différentes mesures de rendement effectuées sur ces deux groupes, il en a été retenu plus particulierement quatre, qui mettent en évidence l'incidence d'une part d'une modification de l'arête de sortie des aubes effectuce sur les indications de M. Fontans: à partir des résultats de l'exploration prealable du champ des vitesses dans l'aspirateur, d'autre part, de la mise en place d'une pointe perforée.

A ces quatre essais correspondent les quatre courbes de la figure 5 :

- Courbe $N^{\circ} 1$ : rendement avec le tracé d'orisine,

coulde $\mathrm{N}^{\circ} 2$ : rendement apris relaille des aubes par le constructeur,

Combe $N^{\circ} 3$ : rendement apres retaille par les soins d'E.D.F.,

Conibe $\mathrm{N}^{\prime \prime}$ t : rendement de celle dernière roue apris pose de la pointe perforée.

Les rendements 1 et 2 ont été mesurés en 1947 par la Division des Essais extérieurs des Etudes al Recherches hydrauliques; les rendements 3 et t en 1954 par la Division Technique générale de la Production hydraulique. Ces courbes ont élé itablies à des époques el avec des moyens diffirents; quoique certains recoupements aient pu être opcrés grâce à une installation permanente de contrôle piézométrique des débits, il a été admis que les rendements ne sont comparables d'une roue à l'autre qu'à $1 \%$ près. La comparaison entre les courbes 3 el 4 est cependant significative à $0,5 \%$ près.

On observera que les courbes 1 et 2 sont relatives à des fonctionnements avec aération, la marche sans air étant instable. Par contre, les courbes 3 et 4 sont relatives à des marches sans aération, devenues normales à la suite des modifications apportées à la roue.

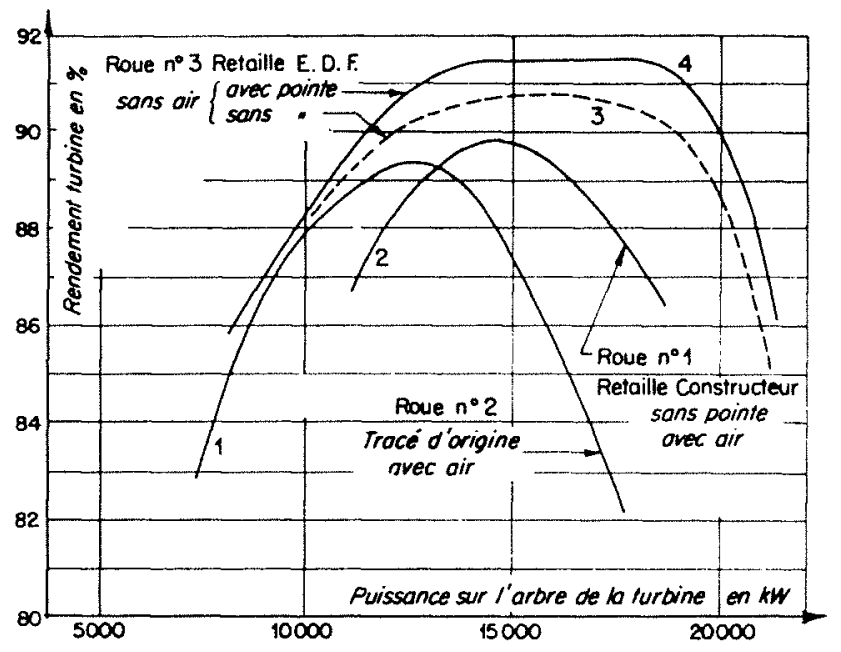

Fig. 5. - Usine de Saint-Geniez-o-Mllelle Essais de decembre 1947 al octobre 1954.

L'examen des graphiques fait ressortir un gain le rendement de l'ordre de 1 point apporté par l'adjonction de la pointe perforée, se superposant a l'élargissement de la zone des hauts rendements et au gain substantiel de puissance (environ $+.000 \mathrm{~kW}$ ) résultant de la retaille.

VIII. - RÉSHLTATS ORTENTS SYR D'AETRES TERBNES

Une pointe perforée a également été posée à Cordéac $(40.000 \mathrm{~kW}, 87 \mathrm{~m}$ de chutc), permeltant la suppression de l'aćration et entrainant la disparition des vibrations et des charges critiques.

Au Poet $(5.000 \mathrm{~kW}, 25 \mathrm{~m}$ de chute), le cône de l'aspiraleur avail pris du jeu dans son seellenent en béton ef se déplaçait d'un $\mathrm{mm}$ a la rharge critique. Il reste tout a fail immobile depuis la pose d'une pointe perforée, sans qu'il ait été nécessaire d'opérer la réfection du scellement. 
Aucune mesure de rendement n'est encore venue compléter ces résultats purement qualitatifs.

Il est vraisemblable d'ailleurs que l'efficacití des pointes perforées se révélera variable suivant le type de turbine et le tracé des aubages; de plus, nous venons de constater sur plusieurs installations que des mises au point s'imposent.

\section{IX. - Conclusions}

Pour conclure, nous croyons utile d'insister sur les points particuliers suivants :

-. L'exploration du champ des vitesses à la sortie de la roue a permis de constater que l'écoulement dans cette zone n'est pas homogène, comme on aurail pu le supposer.

- Des modifications peu importantes permettent souvent d'équilibrer les vitesses de sortie.
- La zone « d'enu vive » étant, dans les cas ètudiés, considérablement étendue vers le centre, il en résulte une répartition différente des angles de sortie qu'on peut être amené à corriger, notamment dans la zone centrale.

-. Enfin les essais effectués font apparaitre pour une même turbine des formes différentes de courbes rendement/puissance utile, mettant en évidence l'importance qu'il y a à ce qu'une turbine soit prévue de telle sorte que :

1" En cas de fonctionnement à chute constante, la surface délimitée par la courbe de rendement pondéré de l'ensemble turbine-conduite-tunnel, soit maximum dans la zone d'utilisation de la machine, ou :

2" En cas de fonctionnement à chute variable, le volume «pondéré », délimité par les courbes en colline, soil maximum dans la zone d'utilisation du groupe.

\section{DISCUSSION}

10 Séance du 10 juin 1954. (Président: M. Huraw)

M. le Président remercie MM. Fontaine et PeYrin de leurs recherches et de leur esprit de collaboration. Il établit un lien entre leur communication et celle de M. Fontren d'après laquelle les pertes de charge sont tres augmentées pal les écoulements de rotation à filets non parallèles; il pense que MM. Fontalve ot Peyrin auraient interet a se mettre en rapport avec $M$. FonTIER, en vue de mettre a sa disposition les moyens lechniques nécessaires a l'application de son étude aux aspirateurs de turbines. D'autre part, II. le Président se demande si, d'après les mêmes résultats de M. Fonrien on ne peut pas esperer qu'au gain de rendemen! de la machine corresponde un gain plus important sur le rendement global de l'installation, en raison du redressement des filets liquides produit dans l'aspirateur et a l'aval de la furbine par lo dispositif de MM. Fontalne et Pevhin.

MM. Fontaine el Perixis confirment at précisent que le dispositif améliore le rendement de l'aspirateur par réduction de la turbulence au sein de l'écoulement ef aussi par réduction des pertes aux parois. Les courbes en colline du laboratoire, qui ont été projetées à l'écran, représentent l'effet sur le rendement global de la turbine à partir de la vanne d'entréce of ce jusqu'à la sortie aval de l'aspirateur.

Le fonctionnement de l'aspirateur intervient évidemment sur l'écoulement de la roue; les surfaces d'écoule. ment définies par les trajectoires méridiennes sont du fait de la réduction du noyau central, ramenées vers les formes et les positions prévues dans le calcul et le dessin de l'aubage; ainsi les sections mouillées des canaux et les angles d'évacuation sont-ils plus proches de ceux qui résultent de l'étude, et il en résulte que le rendement do la roue prise en particulier tend vers le rendement theo. rique ecrit dans les équations du calcul des aubages.

M. Fontaine precise que dans un certain cas la vitesse résiduelle a été réduite de $20 \mathrm{~m} / \mathrm{s}$ à $18 \mathrm{~m} / \mathrm{s}$ en mouillant toute la section du tuyau d'aspiration, ce qui se traduit par une réduction des pertes dans le rapport $182 / 202$.

M. Fontane indique, sur la demande de M. le Prési dent, que le système présenté a été appliqué aux turbines des usines de Chastang, Bort et Saint-Geniez (1) Nerle du Groupe « Dordogne» de l'E.I.F. Dans cette dernière usine, la répartition des vitesses le long des aubes a été complètement modifiéce il en résulte un fain très net dans l'aspirateur; de nourelles mesures globales permettront d'évaluer l'amélioration du fonctionnement de la roue elle-même, dont le rendement itait déjá très bon à la mise en service $(94,6 \%)(1)$.

M. VARlet estime que la communication est plus que savante; elle est extrêmement intéressante en soi. 11 remarque que le procédi revient ì deplacer la zone de vide qui se trouve avant la sortie de la turbine, ce qui se faisait anterieurement par insuffation d'air ef qui, maintenant, consiste a reporter la zone de vide la pointe.

M. Fontaine confirme cette remarque: le dispositif répartit le vide, ainsi que le montre le diagramme des pressions le long d'un rayon de l'aspirateur; au centri on a pratiquement le vide absolu, mais la pression croit très vite. Le but principal est de supprimer l'in" jection d'air qui était un procédé barbare.

En ce qui concerne les ailettes interieures dentraì wement en rotation en masse, les auteurs précisent, sur la demande de M. VanLet, qu'il n'en a pas été exéculées spécialement dans les pointes des turbines de Chastang, le constructeur ayant compté uniquement sur l'entrainement par les brides d'assemblage des deux moitiés de la pointe (conçue en deux pièces pour permettre leur introduction a l'intérieur de l'aspirateur par la porte d'acces) et sur l'entrainement par les très gros ecrous de l'accouplement de la roue à l'arbre, lesquels sont situés en face de la perforation d'éjection.

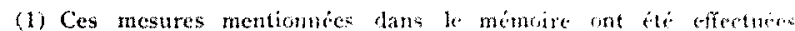
postérieurement î la séance ill 10 juin 1954 , ic relater 
Il est d'ailleurs probable que le seul entrainement par frottement visqueux aux parois suffirait.

La perte de puissance par le pompage, que l'on peut facilement évaluer et qui est très faible, n'atténue pas de façon sensible l'amélioration du rendement obtenue grâce à la pointe perforée.

M. Fontaine indique que les pertes relatives dans le tuyau d'aspiration d'une turbine normale passent par un minimum, puis croissent très rapidement vers la charge maxima. L'intérêt du dispositif d'aspiration consiste à étaler le minimum de ces pertes de manière qu'il se prolonge jusqu'à la zone pratique de fonctionnement.

M. Varlet signale que, dans certaines installations

\section{9" Séance du 25 novembre 195\%. (Président : M. Gariel.)}

Sur la demande de M. le Président, M. Walla précise que l'effet de la «pointe perforée» se tradujt par une amélioration du rendement de un point, mesuré sur la roue $\mathbf{n}^{\circ} 3$ de l'usine de Saint-Geniez-ô-Merle.

M. Fontane ajoute que sur deux autres turbines, l'effet du dispositif n'a pu être chiffré, mais s'est manifesté par leur possibilité de marche sans admission d'air ou avec admission très réduite. Par contre, quelques difficultés se présentent pour l'adaptation de ces dispositifs à une turbine un peu spéciale, en service au Maroc.

il. le Président rappelle les résultats obtenus grâce au dispositif Fontaine-Peyrin (suppression des bruits anormaux, suppression des coups de bélier et régularisation de l'écoulement à l'aval) et souligne le double phénomène de la cavitation et du cheminement du tourbillon, l'une donnant naissance à des bruits dont la fréquence est de l'ordre de la vitesse de rotation et l'autre à de puissants entraînements des tuyaux d'aspiration dans leur logement au rythme de la seconde correspondant, d'ailleurs, dans plusieurs cas observés, à la période propre de l'alternateur. américaines, le moyeu de la roue était prolongé dans le centre de l'aspirateur par un moyeu fixe.

M. PEyrin indique qu'il s'agissait d'aspirateurs c1 général non coudès, du genre Moody; ce matt central était de tenue difficile et précaire et par ailleurs il semble quer l'on n'en ait pas connu exactement les effets sur le rendement. Cette pratique a été abandonnée.

Enfin M. Vanlet attire l'attention sur l'usure de la plaque perforée au bout d'un certain temps de service.

M. Fontaine et M. Perrin estiment que cette usure est, cn effet, inévitable et une fois terminée la période de mise au point du dispositif, qui a nécessité au Chastang, sur le prototype, 27 modifications successives, il pourra être envisagé d'installer une plaque perforée en métal inoxydable susceptible d'un plus long service.

M. Pexrin explique, en s'aidant d'un schéma fait au tableau, qu'à l'intérieur du tuyau d'aspiration, ce tourhillon tourne sur lui-même non pas suivant la loi du vortex, mais suivant une rotation en bloc, qu'il affecte la forme d'un toron hélicoïdal dont le diamètre s'amplific dans la zone de dépression centrale, du fait du dégagement, sous forme de bulles, de gaz dissous, puis qu'il est emporté par le courant ambiant en produisant une détonation dans le tuyau et une intumescence a la sortic aval. Enfin tout se calme, le tourbillon se reforme et disparaît à nouveau quand il a suffisamment grossi. Lc tuyau, sollicité par l'effet centrifuge de la masse de cette torche intérieure, prend un mouvement de balancement rotatif limité par la réaction des scellements mais très visible malgré son amplitude relativement faible. D'autre part, on conçoit qu'une injection d'air insuffisante dans le centre du tuyau réduise la densité du fluide de la torche et augmente donc la fréquence de sa formation. Il est done souhaitable que l'on puisse un jour supprimer complètement cette admission d'air gràce a l'utilisation de pointes perforées.

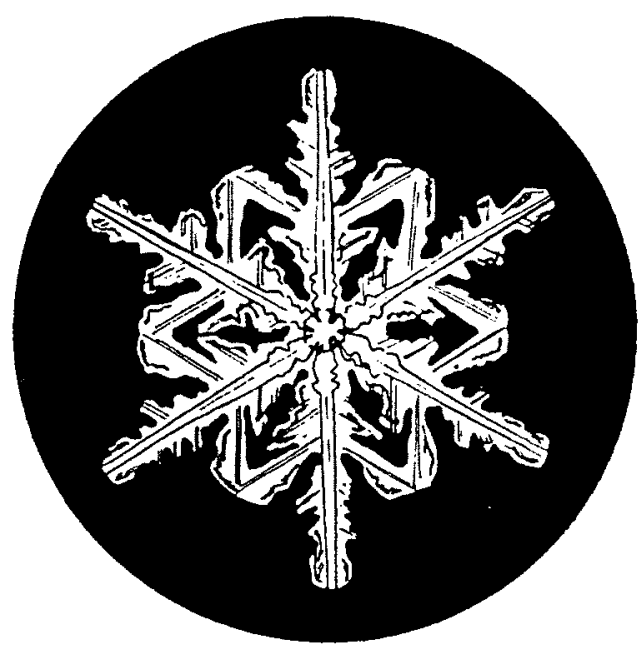

